\title{
Magnetic Anisotropy in GeMnTe - ab initio Calculations
}

\author{
A. ŁusAKOWsKi ${ }^{a}$, P. BogusŁaWsKI ${ }^{a, b}$ \\ ${ }^{a}$ Institute of Physics, Polish Academy of Sciences, al. Lotników 32/46, 02-668 Warszawa, Poland \\ ${ }^{b}$ Institute of Physics, University of Bydgoszcz, J.K. Chodkiewicza 30, 85-072 Bydgoszcz, Poland \\ Density functional theory calculations of the energy of magnetic anisotropy in GeMnTe were performed us- \\ ing OpenMX package with fully relativistic pseudopotentials. The discussion of microscopic origin of magnetic \\ anisotropy was conducted. The main conclusion is that the magnetic anisotropy is caused by interplaty between \\ spin-orbit and Coulomb interactions. The discussion includes also the influence of the free carriers concentration \\ on the amplitude of energy of magnetic anisotropy. The important role of the chemical disorder is also pointed out.
}

DOI: 10.12693/APhysPolA.126.1177

PACS: $75.30 . \mathrm{Gw}, 71.15 . \mathrm{Mb}$

\section{Introduction}

GeTe is a narrow gap IV-VI semiconductor crystallizing at high temperatures in the cubic $\mathrm{NaCl}$ structure. At temperatures below $T_{0}=670 \mathrm{~K}$, a structural transition takes place to the rhombohedral phase with the angle characterizing the unit cell $\alpha \approx 88.2^{\circ}$. In addition, the $\mathrm{Ge}$ and $\mathrm{Te}$ sublattices are displaced relative to each other along [111] direction by a vector $\tau a_{0}(111)$, where the lattice constant $a_{0}=5.979 \AA$ and $\tau \approx 0.03$ [1]. This displacement causes GeTe to be ferroelectric. The above features characterize also $\mathrm{Ge}_{1-x} \mathrm{Mn}_{x} \mathrm{Te}$ mixed crystals, with the difference that the critical temperature $T_{0}(x)$ decreases with the increasing manganese content $x$. Manganese ions introduce in GeMnTe local magnetic moments and this IV-VI diluted magnetic semiconductor exhibits ferromagnetic transition with the Curie temperature $T_{C}(x)$ up to $200 \mathrm{~K}$. The simultaneous presence of ferromagnetic and ferroelectric order (ferroicity) makes this compound very interesting for possible applications.

Recently, magnetization and ferromagnetic resonance (FMR) measurements were performed on monocrystalline layers of $\mathrm{Ge}_{1-x} \mathrm{Mn}_{x} \mathrm{Te}$ grown on $\mathrm{BaF}_{2}$ (111) substrate as well as on polycrystalline, layered $\mathrm{Ge}_{0.9} \mathrm{Mn}_{0.1} \mathrm{Te}$ microstructures [2-5]. The experiments probed the magnetic anisotropy energy in the ferromagnetic state. One of the striking results is that in monocrystalline layers the easy axis of magnetization is perpendicular to the layer while the usual in-plane easy axis due to dipolar interactions (shape anisotropy) is observed in polycrystalline microstructures and is partially recovered in GeMnTe layers upon annealing. The analysis of the angle dependence of the FMR resonance field yielded for $\mathrm{Ge}_{0.85} \mathrm{Mn}_{0.15} \mathrm{Te}$ layer perpendicular magnetic anisotropy field $H_{\mathrm{A}}=0.2 \mathrm{~T}$.

Motivated by the experimental results we decided to study in more detail anisotropy of $\mathrm{Ge}_{1-x} \mathrm{Mn}_{x} \mathrm{Te}$ using density functional theory method. The main aim of the paper is to understand the basic mechanisms responsible for the magnetic anisotropy. The calculations were performed using the open-source OpenMX package for DFT calculations [6] with fully relativistic pseudopotentials. For Mn we used pseudopotentials distributed with the package, for Ge and Te the pseudopotentials were generated using ADPACK, a program distributed with the package.

The important feature of OpenMX package is the possibility to constrain orientations of spins for atoms constituting the supercell. This is done by introducing a properly constructed additional harmonic potential [7] to the Kohn-Sham Hamiltonian. In the following calculations the Mn spin is rotated in (110) plane. Such a rotation corresponds to the situations most frequently studied experimentally when the external magnetic field rotates in this plane in electron ferromagnetic resonace experiment. The angle $\theta$ is measured in this plane from [111] direction and $\theta=90^{\circ}$ corresponds to situation when the Mn spin is along [112] direction.

Another important possibility offered by OpenMX package is the possibility of calculations for crystals structures with nonzero density of free carriers. In the present paper we present results for two hole concentrations $p=0$ and $p=5 \times 10^{21} \mathrm{~cm}^{-3}$.

Because the integrals in general analytical considerations are replaced in numerical calculations by finite sums over discrete sets of points there are two important additional input parameters to the program: the length of the grid in real space and the number of integration points in the unit primitive cell in the reciprocal space (the Brillouin zone). In our calculations the length of the grid in real space is approximately equal to $0.15 \AA$ and it turns out that the further decrease of this length does not change the results significantly. Much more important is the number of integration points in the unit cell in the reciprocal space. This number is not defined generally, it strongly depends on the concentration of holes. In the following pictures we always indicate the number of integration points in the Brillouin zone (BZ).

The energy of magnetic anisotropy is defined as follows. For a assumed set of directions of the manganese spin, after the selfconsistent procedure, we obtain a set of corresponding total system's energies. Next, we calculate the average for this set and the energy of magnetic anisotropy for a given spin's direction is defined 
as the difference between the corresponding system's total energy and this average. In general, if in the formula for the energy of magnetic anisotropy $E_{\mathrm{A}}$ we retain terms up to fourth order in magnetization, the general formula for $E_{\mathrm{A}}$ may be written as $E_{\mathrm{A}}=A \cos 2 \theta+B \sin 2 \theta+$ $C \cos 4 \theta+D \sin 4 \theta+E$. It turns out that for the systems considered in the present paper the angle dependences of the energies of magnetic anisotropy may be described by a simpler formula $E_{\mathrm{A}}=A \cos 2 \theta+E$ with the amplitude $A$ depending on the hole concentration and with the unimportant constant $E$ which has no physical meaning in the present considerations. Such a formula is quite satisfactory for all the presented cases. The other terms become important only when the rhombohedral angle $\alpha$ defining the unit cell is near $90^{\circ}$ and the parameter $\tau \approx 0$, it means in situations when the deviation from perfect cubic symmetry case is very small.

In the following figures, the points correspond to the calculated values and the lines are fits according to the simplest formula $E_{\mathrm{A}}=A \cos 2 \theta+E$.

\section{Results}

All the calculations were performed for $2 \times 2 \times 2 \mathrm{su}-$ percell containing 64 atoms with one Ge replaced with $\mathrm{Mn}$ atom. The replacement of one germanium by manganese atom in such a supercell corresponds to approximately $x=0.03$ in $\mathrm{Ge}_{1-x} \mathrm{Mn}_{x}$ Te mixed crystal. The assumed symmetry of the crystal was rhombohedral with the lattice constant $a_{0}=5.987 \AA, \alpha=88^{\circ}$ and $\tau=0.03$. For such a set of parameters there is well defined energy gap in the electronic band structure thus it is possible to calculate insulating $(p=0)$ case.

In Fig. 1 we compare insulating $(p=0)$ and conducting $\left(p=5 \times 10^{21} \mathrm{~cm}^{-3}\right)$ cases. We see that the amplitude for the conducting case is an order of magnitude larger than in the insulating case. A possible explanation of this fact is proposed below, after the analysis of microscopic mechanism of anisotropy.

Preliminary calculations, not reported in the present paper, show that the changes in hole concentration influence not only the value of the anisotropy energy but may also lead to the change of direction of the easy axis of magnetization.

Figure 1 also shows that the convergence, in the sense of the number of integration points in the Brillouin zone, in the insulating case is much faster than in the conducting case. For $p=0$ the curve for 512 integration points in BZ is not shown because it is practically identical to that for 343 points. Contrary, for the conducting case the convergence is much slower. The results cannot be taken as the quantitative, we may only speak about the direction of the easy axis of magnetization.

In order to better understand the mechanism of anisotropy in $\mathrm{Ge}_{1-x} \mathrm{Mn}_{x} \mathrm{Te}$, in Fig. 2 we present the contributions to the energy of magnetic anisotropy $E_{\mathrm{kin}}$, $E_{\text {coul }}$ and $E_{\mathrm{xc}}$ resulting from the kinetic, Coulomb and exchange-correlation energies, respectively. These three contributions are calculated from the respective energies
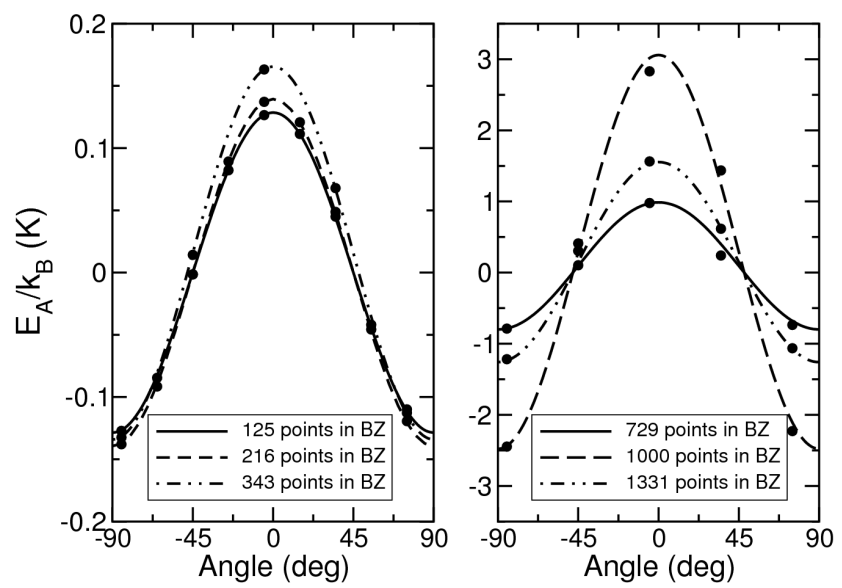

Fig. 1. Angle dependence of magnetic anisotropy energy for $2 \times 2 \times 2$ supercell for $a_{0}=5.987 \AA, \alpha=88^{\circ}$ and $\tau=0.03$ for insulating (left part) and conducting (right part) cases. Points - results of calculations, lines - fits.
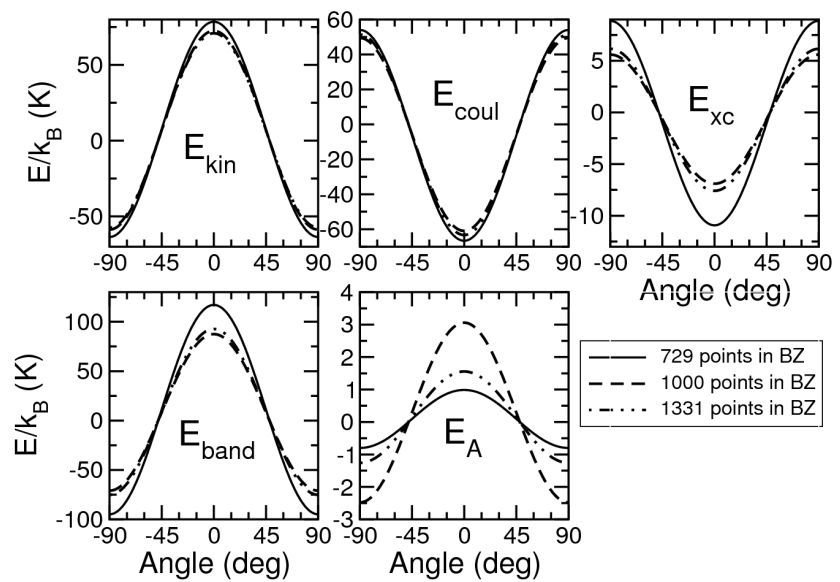

Fig. 2. Angle dependence of magnetic anisotropy energy components for $2 \times 2 \times 2$ supercell for $p=5 \times$ $10^{21} \mathrm{~cm}^{-3}, a_{0}=5.987 \AA, \alpha=88^{\circ}$ and $\tau=0.03$.
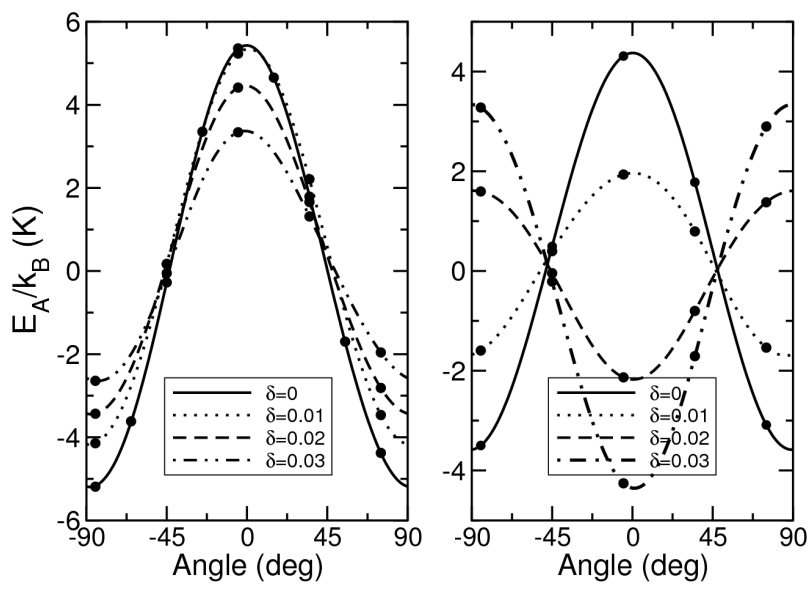

Fig. 3. Angle dependence of magnetic anisotropy energy components for $2 \times 2 \times 2$ supercell for $p=5 \times$ $10^{21} \mathrm{~cm}^{-3}, a_{0}=5.987 \AA, \alpha=88^{\circ}$ and $\tau=0.03$ for different positions of $\mathrm{Mn}$ atom (left) and different positions of nearest Te atoms (right). 
in the same way as $E_{\mathrm{A}}$ from the total energy of the supercell and when summed give the energy of anisotropy $E_{\mathrm{A}}$. In the figure we also present the band energy $E_{\text {band }}$ resulting from the sum, over occupied states, of eigenvalues of Kohn-Sham Hamiltonian. Let us notice that the Coulomb energy contains not only electron-electron interaction, but also the interaction of electrons with the cores, thus it takes into account also spin-orbit interaction because the pseudopotentials are spin-dependent. From analysis of Fig. 2 we draw conclusion that changes of manganese spin direction results in significant changes of electron density. This is caused by the presence of spin-orbit interaction. For each direction of manganese spin the electron density assumes a certain distribution which leads to a minimum of total energy. Then the change of direction of $\mathrm{Mn}$ spins, due to spin-orbit interaction, causes an increase of the total energy of the system. This increase is partially compensated by the redistribution of the electron charge. In other words, one may say that the magnetic anisotropy energy is a result of the interplay between the spin-orbit and Coulomb interactions. Let us also notice that the angular changes of $E_{\text {kin }}, E_{\text {coul }}$ and $E_{\mathrm{xc}}$ are much larger than the changes of their sum.

If we accept this point of view we may propose a qualitative explanation for the difference of magnitude of magnetic anisotropy energy between insulating and conducting cases. The manganese spin polarizes the neighbouring atoms. As we checked the directions of Ge spins are always parallel to the direction of $\mathrm{Mn}$ spin and opposite to the Te spins. This polarization of neighboring spins extends on much larger distances in the conducting case. Consequently, the changes of the direction of Mn spin result in larger changes in spin-orbit energies and larger redistribution of charge.

Finally, let us point an attention to the fact that the behavior of the magnetic anisotropy energy is extremally sensitive to the local crystal neighbourhood of manganese ion. According to the definition of $\tau$, the Te sublattice is displaced with respect to the Ge sublattice along [111] crystal direction by $a_{0} \sqrt{3} \tau$. In calculations, the results of which are presented in Fig. 3, in the left part the Mn ion is moved by $a_{0} \sqrt{3} \delta$ and in the right part the six nearest tellurium neighbours of Mn ion are displaced not by $a_{0} \sqrt{3} \tau$ but only by $a_{0} \sqrt{3}(\tau-\delta)$. Of course, the symmetry of the nearest neighbourhood of $\mathrm{Mn}$ ions is always rhombohedral, because $\alpha$ is not equal to $90^{\circ}$. We see the significant influence of the nearest neighbourhood on the magnetic anisotropy energy, not only on the value, but even when the perturbation of the crystal lattice is larger, on the direction of the easy axis of magnetization.
In real mixed crystals we always have local crystal lattice deformations caused by chemical disorder which is due to random placement of atoms of different species and different ionic radii. The results presented in Fig. 3 clearly show that the model aiming in obtaining quantitative results for real crystals, certainly must take disorder into account.

\section{Conclusions}

The main conclusions are following:

1. Despite very small values of magnetic anisotropy energy it is possible to calculate this quantity or at least to predict the direction of the easy axis of magnetization.

2. The direction of the easy axis of magnetization and the value of the energy of anisotropy depend on the hole concentration.

3. The changes of the magnetic ion spin direction result in significant redistribution of the electron density. From the microscopic point of view the magnetic anisotropy is an interplay between spin-orbit and Coulomb interactions.

4. Even small changes in the atoms' positions result in noticeable changes in the values of magnetic anisotropy. Thus the model of the anisotropy certainly must take into account the chemical disorder inevitable in the real crystals.

\section{Acknowledgments}

The authors acknowledge the support from NCN (Poland) research project no. UMO2011/01/B/ST3/02486.

\section{References}

[1] Y. Fukuma, T. Murakami, H. Asada, T. Koyanagi, Physica E 10, 273 (2001).

[2] W. Knoff, P. Dziawa, V. Osinniy, B. Taliashvili, V. Domuchovski, E. Łusakowska, K. Świątek, T. Story Mater. Sci.-Poland 25, 295 (2007).

[3] W. Knoff, K. Świątek, T. Andrearczyk, V. Domukhovski, P. Dziawa, L. Kowalczyk, E. Łusakowska, A. Siusys, B. Taliashvili, J. Wróbel, T. Story Phys. Status Solidi B 248, 1605 (2011).

[4] Y. Fukuma, H. Asada, S. Miyawaki, T. Koyanagi, S. Senba, K. Goto, H. Sato Appl. Phys. Lett. 93, 252502 (2008).

[5] H. Przybylińska, G. Springholz, R.T. Lechner, M. Hassan, M. Wegscheider, W. Jantsch, G. Bauer, Phys. Rev. Lett. 112, 047202 (2014).

[6] See http://openmx-square.org.

[7] See http://openmx-square.org/tech_notes/tech_ notes.html. 